\title{
Stress Quantification using Fuzzy Analysis of ECG Parameters
}

\author{
Sneha Mittal \\ ECE Dept., \\ GNE College \\ Ludhiana, Punjab, India
}

\author{
Nirmal Singh Grewal, Ph.D \\ ECE Dept., GNE College \\ Ludhiana, Punjab, India
}

\begin{abstract}
Mental stress quantification using fuzzy analysis of ecg parameters is presented here. ECG signal is decomposed using the BIOR-3.9 wavelet family upto three levels. The approximates signals are used for computation ecg parameters like energy, entropy, power, standard deviation, mean and covariance. A fuzzy classifier is designed using trimf function as associate membership in fuzzy analysis. The ecg data base is taken from MIT data base web site.
\end{abstract}

\section{Keywords}

ECG, BIOR-3.9 wavelet, Entropy, Energy, Power, Standard Deviation, Covariance, Fuzzy Logic, Mental Stress

\section{INTRODUCTION}

The stress assessment is now-a-days a very common need for the peoples who are extra busy in their daily life. Also, the life is becoming more and more busy due to fast moving era. Therefore, stress/pressure on mind is quite inevitable. To cop up with the mental stress, it is first to measure or quantify the stress level so that a proper cure could be initiated. However, for a person responding to the outside world, it is easier to measure the stress and cure for the same. But for a person who is in coma or can not respond, in that case it becomes a tedious task to determine the stress level and cure for the same.

There is previous research in the direction of mental stress assessment. It has observed that the research leads to qualitative model. That is, the model present the state of mind that whether the person is stressed or not stressed. This gives an incomplete picture of the person under scanner. If the same attribute is made quantitative, then the treatment may be more calculated and the response of the treatment may also in turn be quantitative. The presented work is based on this assumption that the stress could be numerically quantified and labeled according to its numerical figure.

\section{RELATED WORKS}

Fetal ECG (FECG) tele-monitoring is an important branch in telemedicine. The design of a tele-monitoring system via a wireless body-area network with low energy consumption for ambulatory use is highly desirable. [1].

In some cases Wearable ECG sensors can assist in prolonged monitoring of cardiac patients. In this paper, compressed sensing is employed in ECG compression. [2].

A wavelet-based method for the compression of electrocardiogram (ECG) signal is also prepared. The discrete wavelet transform (DWT) is applied to the digitized ECG signal; then the resulting wavelet coefficients are threshold using a threshold based on energy packing efficiency of the wavelet coefficients. [3].
Emotional stress refers to the inducement of stress due to the consequence of a continuous experience of negative emotions (sad, anger, fear and disgust). This work aims to investigate the effect of negative emotions in emotional stress inducement through Electrocardiogram (ECG).

Self Assessment Manikin (SAM) and Self Assessment Form is used to evaluate the subject emotional response during and after the data acquisition, respectively. Statistical features such as heart rate (HR), approximate entropy (Ap En), mean R amplitude (MRA), mean R-R interval (MRRI), standard deviation of normal to normal RR intervals (SDNN) and root mean square of successive heartbeat interval differences (RMSSD) are extracted from HRV signals.[4]

QRS complex is analyzed using the geometrical feature extraction algorithm. A fuzzy classifier is presented for computing the heart rate of the person under test[5]

Heart rate variability is assesses and a correlation is established between HRV and mental stress. A fuzzy clustering is also proposed for clustering of different data leading to construction of mental stress computation[6].

A fourier transform based approach is used to analyse the ecg signal and draw the conclusion for computation of mental stress. [7]

A wavelet approach is presented for analysis of ecg signal and denoising of the same. The denoised ecg signal is then exposed to stress assessment algorithm. [8].

\section{METHODOLOGY}

ECG data base used in the presented work is based on MIT data base. Stressed and normal ECGs are taken from the MIT data base directory web site for testing and validation purposes. The ecg is decomposed using BIOR-3.9 wavelet family upto three levels and the approximate signal is used for computation of ecg parameters like energy, entropy, power, standard deviation, mean and covariance. The parameters are defined as follows:

$$
\begin{gathered}
\text { Energy }(E)=\sum_{i=1}^{n} x_{i}^{2} \\
\text { Power }(P)=\frac{1}{n} \sum_{i=1}^{n} x_{i}^{2} \\
\text { Mean }(\mu)=\frac{1}{n} \sum_{i=1}^{n n} x_{i} \\
\text { Entropy }(\text { Ent })=-\sum_{i=1}^{n} p(x i) \cdot \log _{10} p(x i) \\
S D=\frac{1}{n} \sum_{i=1}^{n}\left(x_{i}-\mu\right)^{2}
\end{gathered}
$$




\section{ALGORITHM}

Membership Function Used: Triangular-shaped membership function

built-in

\section{Matlab Function $\rightarrow$ trimf}

The triangular curve is a function of a vector, ' $\mathrm{x}$ ', and depends on three scalar parameters ' $a$ ', 'b', and 'c', as given by:

$$
f(x ; a, b, c)=\left\{\begin{array}{cc}
0, & x \leq a \\
\frac{x-a}{b-a}, & a \leq x \leq b \\
\frac{c-x}{c-b}, & b \leq x \leq c \\
0, & c \leq x
\end{array}\right\}
$$

or, more compactly, by :

$$
f(x ; a, b, c)=\max \left(\min \left(\frac{x-a}{b-a}, \frac{c-x}{c-b}\right), o\right)
$$

The parameters ' $a$ ' and ' $c$ ' locate the "feet" of the triangle and the parameter $\mathrm{c}$ locates the peak.

The membership functions for the energy and entropy values Vs Stress for ecg are shown below:

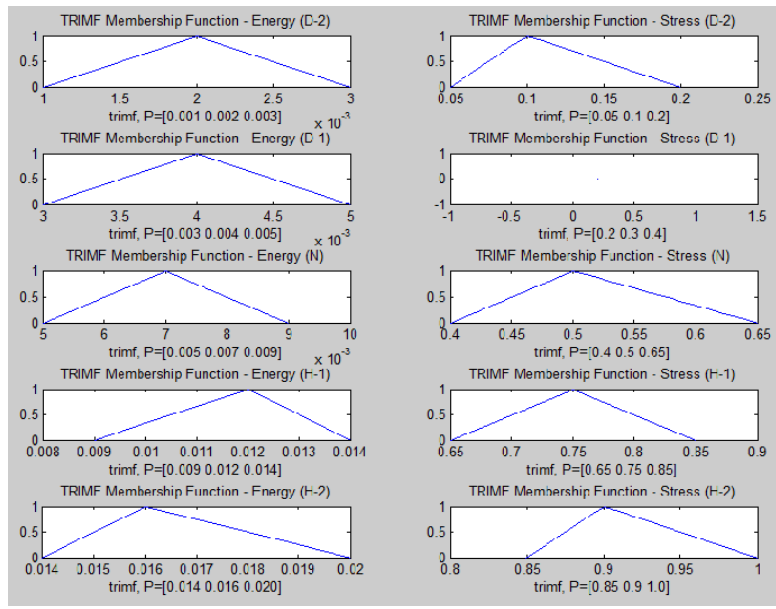

Energy Vs Stress Fuzzy Membership Function

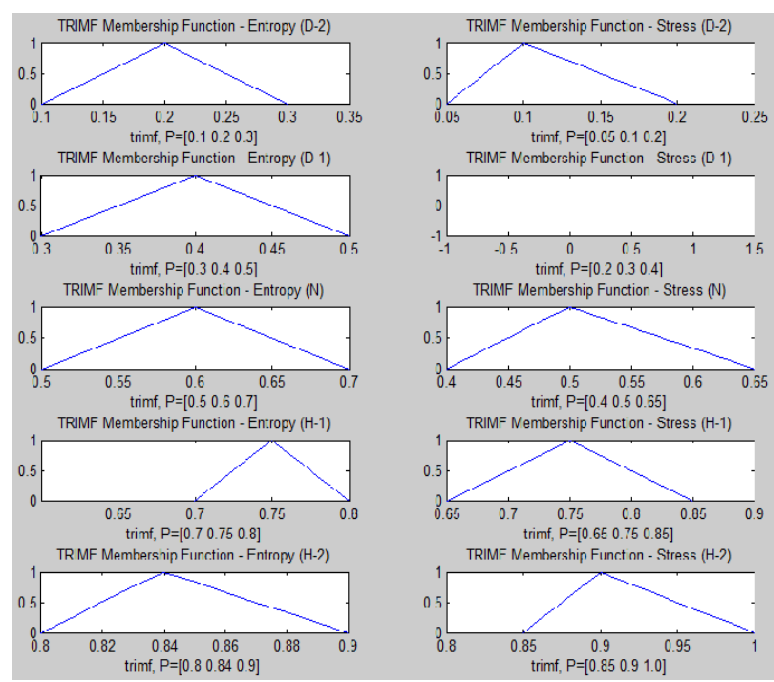

Entropy Vs Stress Fuzzy Membership Function
Stress Computation using Fuzzy Logic Membership Functions

Rule Base:

Rule \# 1: if( Energy is V. Low AND Entropy is V. Low),

Then Stress case $\rightarrow$ D-2

Rule \# 2: if( Energy is Low AND Entropy is Low),

Then Stress case $\rightarrow$ D-1

Rule \# 3: if(Energy is Normal AND Entropy is Normal),

Then Stress case $\rightarrow \mathrm{N}$

Rule \# 4: if( Energy is High AND Entropy is High),

Then Stress case $\rightarrow$ H-1

Rule \# 5: if( Energy is V. High AND Entropy is V. High),

Then Stress case $\rightarrow$ H-2

\section{RESULTS}

Case-1

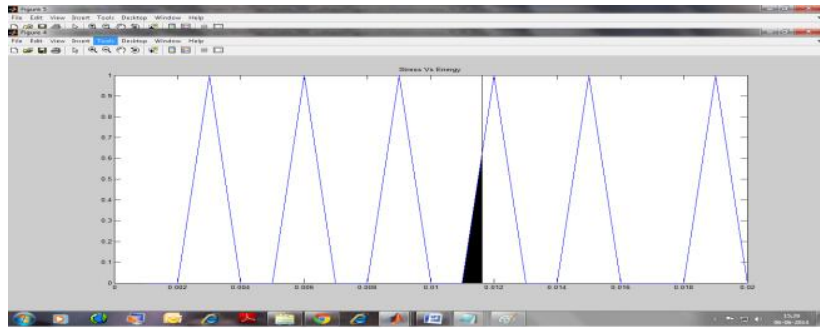

Energy Plot of Case-1

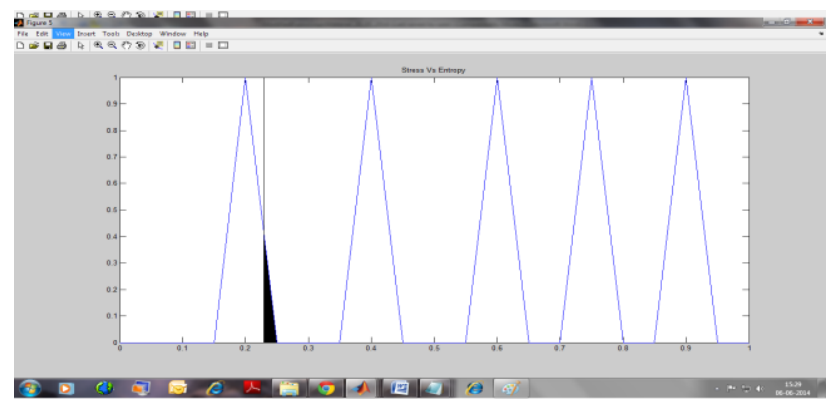

Entropy Plot of Case-1

ECG Analysis

File Path = F:\Project_Sneha_GNE,

LudhianalProgramslfinal_normal_ecg_6.TXT

Date: 6- 6-2014 Time: 15-34-36

Data Base Type --> MIT

ECG Data Length $=4960$

Feature Vector Set at Level- 0

Power Energy Mean Std. Dev. Entropy CV

$\begin{array}{llllll}0.000 & 0.002 & -0.000 & 0.001 & 0.161 & 0.000\end{array}$

Feature Vector Set at Level- 1

Power Energy Mean Std. Dev. Entropy CV

$\begin{array}{llllll}0.000 & 0.002 & -0.000 & 0.001 & 0.208 & 0.000\end{array}$

Final Stress $=0.602511$

ECG --> Normal Case 
Case-2

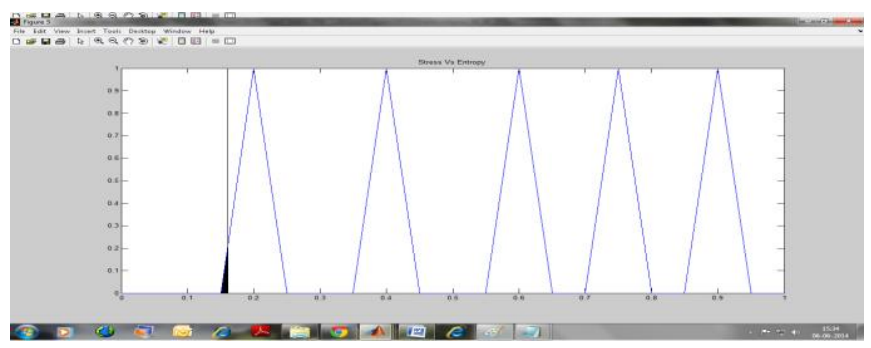

Energy Plot of Case-2

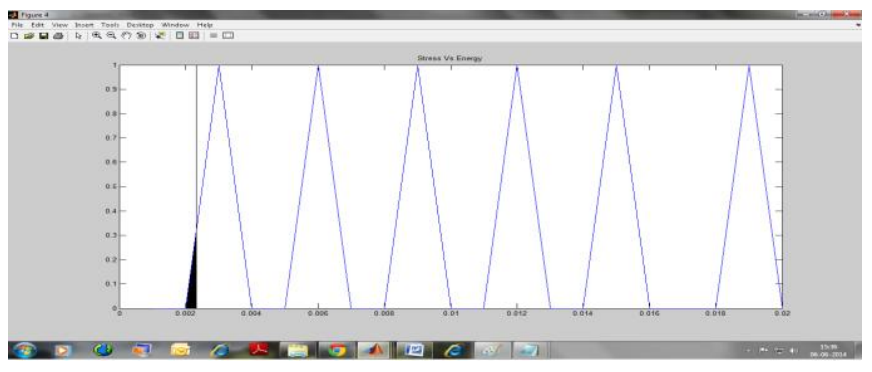

Entropy Plot of Case-2

File Path = F: $\backslash$ Project

LudhianalProgramslfinal_stress_ecg_2.TXT

Date: 6- 6-2014 Time: 15-31-44

Data Base Type --> MIT

ECG Data Length $=4960$

Feature Vector Set at Level- 0

Power Energy Mean Std. Dev Entropy CV

$\begin{array}{llllll}0.000 & 0.012 & -0.001 & 0.001 & 0.229 & 0.000\end{array}$

Feature Vector Set at Level- 1

Power Energy Mean Std. Dev. Entropy CV

$\begin{array}{llllll}0.000 & 0.012 & -0.001 & 0.002 & 0.362 & 0.000\end{array}$

Final Stress $=1.265727$

ECG --> Hyper Case - 2

\section{CONCLUSION}

The presented algorithm has been tested and validated on MIT data base of ecg under normal and stressed conditions The results trends show a fair correlation with medical symptoms of the stress and normal condition persons. It is observed from the results that energy and entropy play a significant role in deciding the stress level. These two are dominant factor in the assessment of mental stress. More work could be done on improving the computation of entropy and energy of the ecg signal so as to authenticate the mental stress computed from the same.

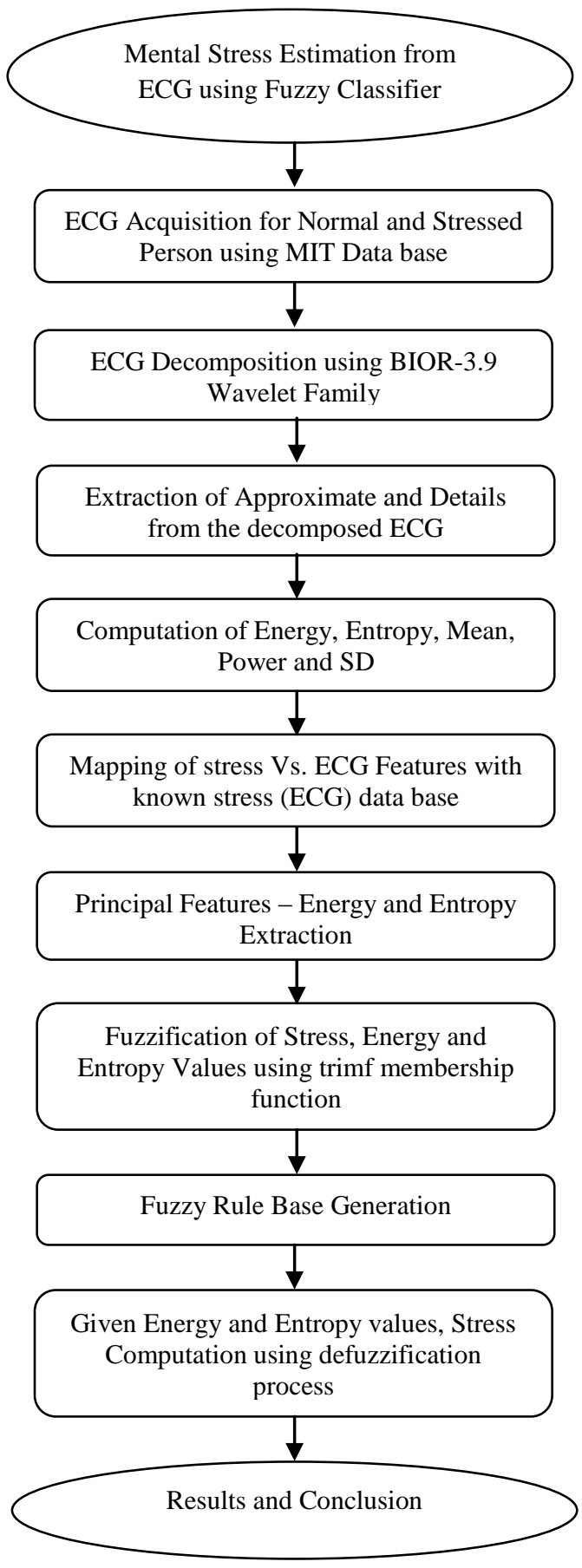

Flow Chart of the System 


\section{REFERENCES}

[1] Zhilin Zhang_, Student Member, IEEE, Tzyy-Ping Jung, "Compressed Sensing for Energy-Efficient Wireless Telemonitoring of Non-Invasive Fetal ECG via Block Sparse Bayesian Learning", ACCEPTED BY IEEE TRANSACTIONS ON BIOMEDICAL ENGINEERING, 2012

[2] Fahimeh Ansari-Ram, Saied Hosseini-Khayat, "ECG Signal Compression Using Compressed Sensing with Nonuniform Binary Matrices", 978-1-4673-1479-4/12/\$31.00 @2012 IEEE

[3] M. Sharafat Hossain , "ECG Signal Compression using Energy Compaction Based Thresholding of the Wavelet Coefficients", DUET Journal Vol. 1, Issue 2, June 2011.

[4] Bong Siao Zheng, M Murugappan and Sazali Yaacob, "FCM Clustering of Emotional Stress using ECG Features", International conference on Communication and Signal Processing, April 3-5, 2013, India.

[5] Mohammad Reza Homaeinezhad1, 2, Ehsan Tavakkoli1,2, Ali Ghaffari, "Discrete Wavelet-based Fuzzy Network Architecture for ECG Rhythm-Type Recognition: Feature Extraction and Clustering- Oriented Tuning of Fuzzy
Inference System" , International Journal of Signal Processing, Image Processing and Pattern Recognition Vol. 4, No. 3, September, 2011.

[6] Mohit Kumar, Matthias Weippert, Reinhard Vilbrandt, Steffi Kreuzfeld, and Regina Stoll, "Fuzzy Evaluation of Heart Rate Signals for Mental Stress Assessment" , IEEE TRANSACTIONS ON FUZZY SYSTEMS, VOL. 15, NO. 5, OCTOBER 2007.

[7] G.Ranganathan, V.Bindhu, Dr.R.Rangarajan, s "ECG Signal Processing using Dyadic wavelet for Mental Stress Assessment", 978-1-4244-4713-8/10/\$25.00 @2010 IEEE.

[8] C. Saritha, V. Sukanya, Y. Narasimha Murthy, "ECG Signal Analysis Using Wavelet Transforms", Bulg. J. Phys. 35 (2008) 68-77. Prof, Shamla Mantri, Dr. Pankaj Agrawal, Prof. Dipti Patil, Dr. V. M. Wadhai

[9] “Depression Analysis using ECG Signal”, ISSN 2277-3061, $\mathrm{N}$ ov 10,2013

\section{AUTHOR'S PROFILE}

Sneha Mittal has received the B.Tech. Degree in EIE from,IITT POJEWAL, Punjab in 2005 and pursuing his M.Tech in ECE from GNDEC Ludhiana, Punjab.. Her field of interest is in Bio medical based application system developments 